\title{
Urban traffic analysis from a large scale floating car data system
}

\author{
F. A. Pugliese, G. Montesano \& G. Valenti \\ ENEA, Italian National Agency for New Technologies, \\ Energy and the Environment, Italy
}

\begin{abstract}
The actual knowledge of traffic performances and travel patterns existing in an urban area is crucial to quantify the effects of transportation improvements and of actions that have to be pursued to improve mobility, as well as even an essential input to calibrate land use and traffic simulation models.

In this view the use of Floating-Car Data (FCD) is emerging as a reliable and cost-effective way to gather accurate traffic data for a wide-area road network and to improve the analysis of travel conditions.

The purpose of this paper is to provide an insight into the potential application of wide scale FCD for improving the traffic analysis process.

The reliability of traffic estimates from FCD largely depends not only on the accuracy but also on the size of the input data: until now FCD experiments have been carried out with few equipped vehicles, maximum some dozen; moreover, mostly of them were special vehicles like taxi or bus, with preferential routes, so their behaviour cannot be taken as general reference.

The originality of this study lies in the large number of private-owned cars involved corresponding to a penetration rate of about 1.3 percent.

A full day of FCD related to the city of Florence is examined in this paper to estimate some travel related measures that cannot be readily replicated on a dayby-day basis using other data sources such as user surveys.
\end{abstract}

Keywords: floating car data, traffic analysis, traffic management.

\section{Introduction}

The use of real-time Floating-Car Data (FCD), based on traces of GPS positions, is emerging as a reliable and cost-effective way to gather accurate traffic data for 
a wide-area road network and also to improve short-term predictions of travel conditions.

Unlike other traffic data collection techniques (Automated Vehicle Identification systems, video cameras, inductive loops, radar based sensors, etc.), floating cars, using GPS receiver and GSM/GPRS transmitter, act as moving sensors travelling in a traffic stream and do not require instrumentation to be set up on the roadway.

Besides increasing the understanding of individual travel behavior, floating car technique can easily provide near real-time traffic performance data for any part of large networks and can offer a viable way to complement fixed-point traffic sensors, avoiding high installation and maintenance cost.

The FCD technique is based on the exchange of information between a fleet of floating cars travelling on a road network and a central data system. The floating cars periodically send the recent accumulated data on their position (latitude, longitude and altitude) and, optionally, instantaneous speed. The frequency of sending/reporting is usually determined by the resolution of data required and by the method of communication between vehicle and data center.

The reliability of travel time estimates based on FCD highly depends on the percentage of floating cars participating in the traffic flow [1-3]; other factors affecting the reliability of travel time estimates, mainly for lower penetration of floating cars, are traffic conditions and road link capacities. As a rule, a lower percentage of floating cars is required in more congested traffic condition, while a higher percentage of floating cars is needed in low flow conditions.

The most useful information that FCD technique provides is average travel times and speeds along road links or paths [4-6]. Until now, various approaches have been proposed to deploy FCD in order to predict short-term travel conditions, to detect incident or critical situations [7-9] and, finally, to determine Origin-Destination traffic flow patterns [10].

This paper is focused on the use of analytical techniques to transform raw FCD into useful information, so that a full understanding of traffic and travel patterns existing in an urban area can be gained. Unlike previous FCD analysis mostly using data from taxi or bus fleets, the FCD used in this study relies on a large fleet of privately owned vehicles [11].

A full day of FCD related to the city of Florence is examined and discussed to demonstrate the practical potential of this approach in the day-to-day monitoring of urban traffic performance.

The paper is organized as follows. Section 2 provides a brief outline of the FCD system and an overview of the case study area. Section 3 presents the main outcomes of the study including some key performance measures for vehicular traffic in the urban area of Florence. Section 4 concludes this paper.

\section{Case study description}

This study uses data from Octotelematics FCD system. Octotelematics [11] is the European leader for development and deployment of Telematics for Insurance application, with approximately 700.000 On Board Units (OBU) installed at the 
time of writing this paper, and provides solutions from in-vehicle On Board Units up to Data Processing Center.

The OBU consists of a GPS receiver, a GPRS transmitter, a 3-axis accelerometer sensor, a battery pack, a mass memory, and a processor/RAM. The OBU stores GPS measurements and periodically transmits (on request or automatically) the recent accumulated measurements to the central data system.

Each record of the car tracking data set includes: a) car identification number (ID), b) Log of date and time in GMT (Greenwich Meridian Time), c) Longitude and latitude of GPS track, d) Speed of the vehicle from GPS, e) course-angle at which the equipped car is travelling with reference to the North, f) GPS status, indicating the measurement accuracy that depends on how many satellites are visible, g) engine status ("starting", "in motion" and "turning off"), h) the actual distance traveled from the previous record.

The area in which analysis has been conducted is the city of Florence, Italy. It has approximately 366.000 inhabitants and covers an area of $141 \mathrm{~km}^{2}$ [12]. It is the economic and administrative capital of the region Tuscany.

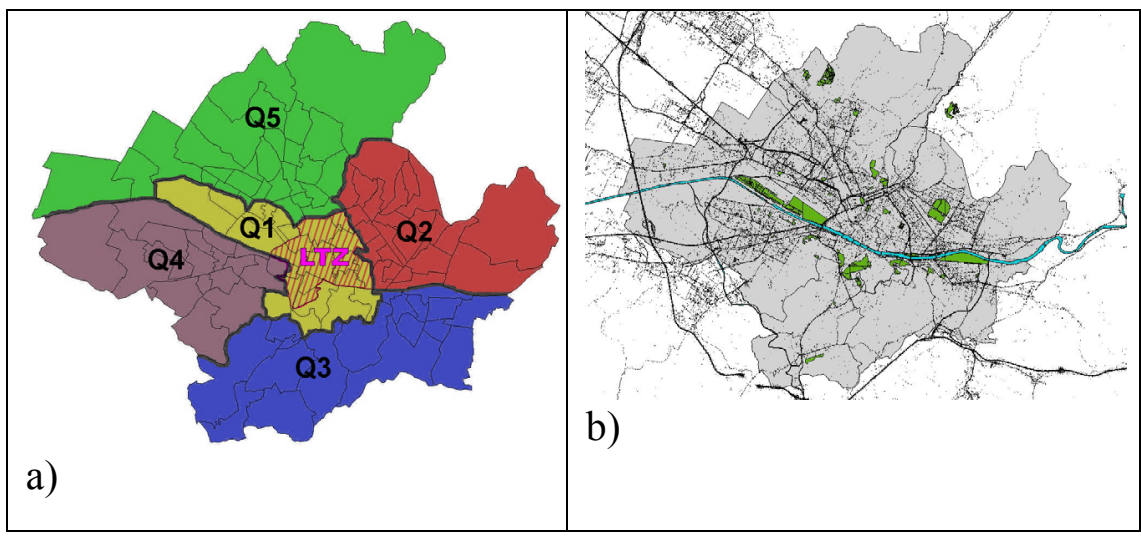

Figure 1: a) Quarters of the municipality of Florence; b) map of the gathered GPS points.

The case study area is the municipality of Florence currently divided into 5 quarters as depicted in Fig. 1.a; The outcomes of this study are both referred to the whole municipality and the quarter "Q1", which includes the ancient historical centre and some adjacent residential zones [13, 14]. Part of the historical centre is currently closed to the vehicular traffic, except buses, taxi and residents with a special authorization. This Limited Traffic Zone (LTZ) is controlled by an Automated Access Control System and traffic restrictions are currently on weekdays from 7.30 a.m. to 7.30 p.m. and on Saturday from 7.30 a.m. to 6.00 p.m.[13].

FCD related to the whole day of Tuesday 4 March 2008 are used in this study. On this date the penetration rate of floating vehicles was estimated by Octotelematics at about 1.3 percent. All the travels having an origin and/or a 
destination within the study area (Internal-Internal, Internal-External and External-Internal), as well as all the through trips, with both the origin and destination lying outside the study zone (External-Internal), have been considered and examined. In fig. 1.b the daily GPS positions, stored by all the floating vehicles travelling in the study area, are displayed on the map.

\section{Travel patterns and traffic performance measures}

An analysis of errors was carried out before transforming raw FCD into travel data. A travel is given by the sequence of GPS locations recorded from the engine "switch on" status to the next engine "switch off" status.

FCD correction and travel reconstruction procedures have been applied to identify possible measurement and recording errors and to complete travel data affected by the lack of data transmission and/or adequate satellite coverage.

From this preliminary work, a total number of 14719 travels performed by 4002 vehicles were identified and analysed. In the full day, each floating vehicle averaged 3.6 travels, a total travel distance of 14,5 kilometres and a total travel time of about 36 minutes.

In the municipality area of Florence the average travel time was about 10 minutes, the average travel distance was about 4 kilometres and the average travel speed was about $24 \mathrm{~km} / \mathrm{h}$. The average travel speed is the total distance travelled divided by the total time taken to travel by the fleet of floating vehicles.

The analysis of FCD revealed that 50 percent and 80 percent of the travels performed by the floating vehicles lasted, respectively, lower than about 9 minutes and 16 minutes (Fig. 2). Moreover about 50 percent and 70 percent of the travels were respectively lower than 3 kilometres and 5 kilometres (Fig. 3).

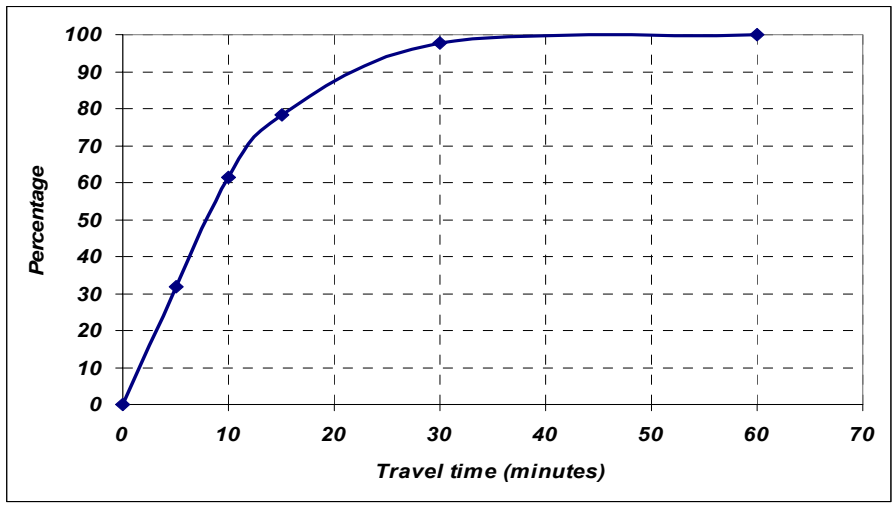

Figure 2: Cumulative distribution of travel times.

Moreover the analysis of the daily travels performed by floating vehicles showed that:

- about 73.5 percent (10824 travels) had both its origin and destination inside the study area; 
- about 23.5 percent (3451 travels) had either its origin or destination outside the study area;

- about 3 percent (444 travels) had both origin and destination outside the study area, but they went through the city.

FCD time logs, as well as following figures, are in GMT, but local time is GMT+1. Fig. 4 depicts the 24-hour profile of generated and attracted travels in the whole study area.

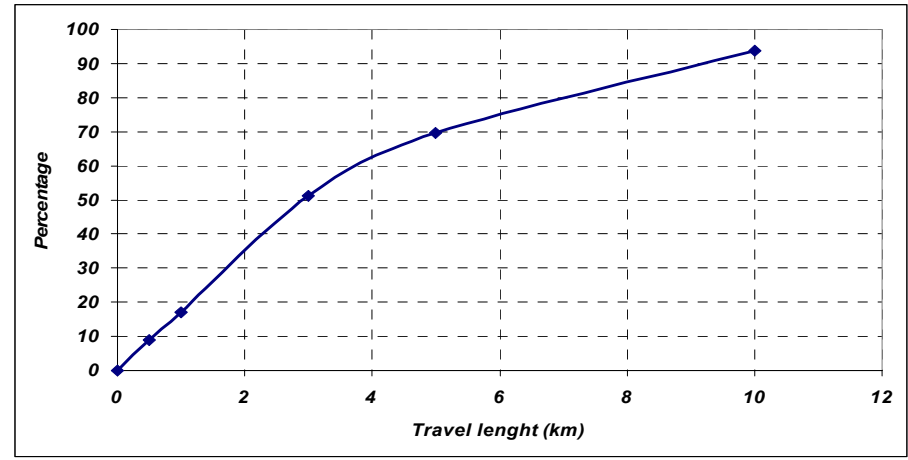

Figure 3: Cumulative distribution of travel distances.

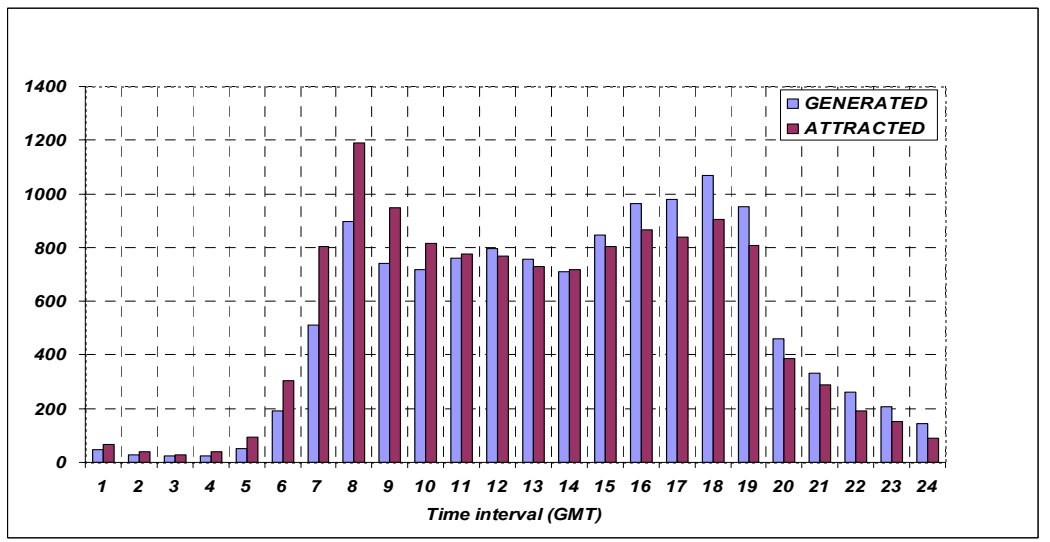

Figure 4: 24-hour profile of travels generated and attracted in Florence.

The higher volumes of generated travels occurred from 4:00 to 7:00 p.m. (from 5.00 to 8.00 p.m. local time) whereas the higher volumes of attracted travels occurred from 7:00 to 9:00 a.m. (from 8.00 to 10.00 p.m. local time). It should be noted that in the first half part of the day travels coming from outside the study area (External-Internal) significantly contributed to the high values of attracted. 
In Fig. 5 the graphs of the average speed respectively for the whole study area and the quarter "Q1" are reported, starting from 6.00 a.m. (GMT) when the number of travels becomes prominent, as shown in Fig. 4. Significant lower speed was estimated in the central quarter "Q1": from 7:00 a.m. to 8:00 p.m. (from 8.00 a.m. to 9.00 a.m. local time) the average speed estimated in the quarter "Q1" varied from a minimum of 14 to a maximum of $17 \mathrm{~km} / \mathrm{h}$ whereas in the whole study area the average speed ranged from a minimum of 21 to a maximum of $27 \mathrm{~km} / \mathrm{h}$.

Furthermore, considering the whole study area, the average speed in rush hour, around 8.00 a.m. and 16.00 p.m. (9.00 a.m. and 17.00 p.m. local time) is

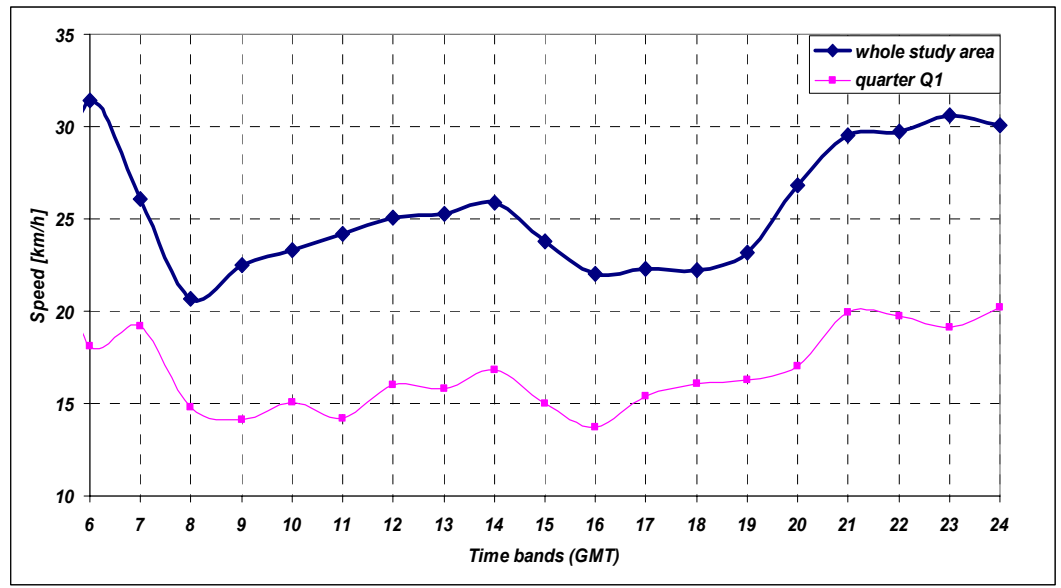

Figure 5: Graphs of average travel speed.

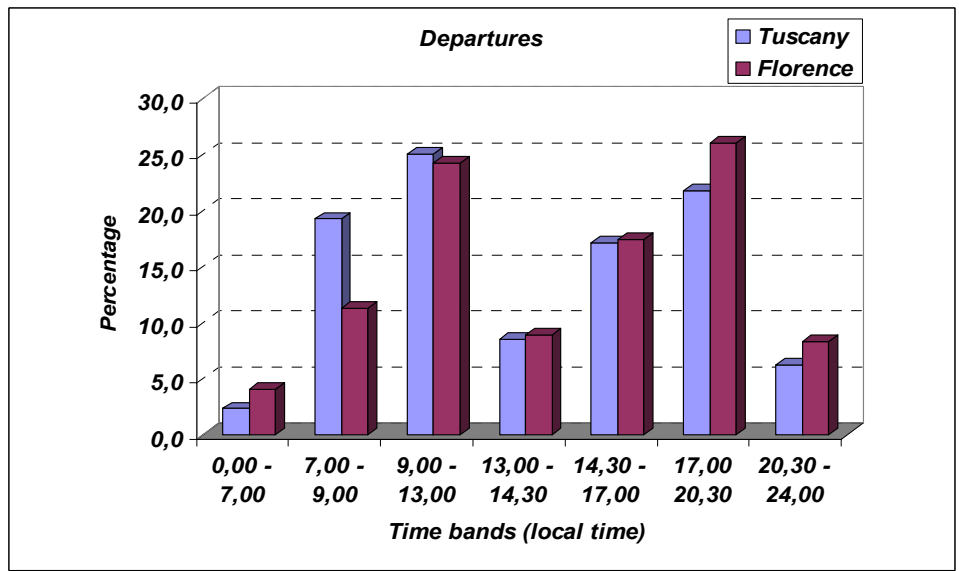

Figure 6: Comparison of historical data (Tuscany) and FCD (Florence). 
about $30 \%$ lower than speed in off-peak hours, around 6.00 a.m. and 11.00 p.m. (7.00 a.m. and midnight, local time).

Travel data of floating vehicles were then compared with previous travel data obtained with traditional surveys carried out by AUDIMOB ("Observation Agency for mobility style and behaviour of Italian people") for the whole Tuscany [15]. Fig. 6 gives a comparison of the volumes of generated travels according to 7 time bands and shows a rather good accordance between them.

Some relevant difference in percentage of departures in time band from 7.00 to 9.00 a.m. can be observed, due to the fact that mostly in this interval the urban area of Florence attracts traffic from outside, as depicted in Fig. 4, so the percentage for all the region is obviously higher.

Finally, an analysis of travels performed by the floating vehicles in the Limited Traffic Zone was carried out.

Fig. 7 shows the 24-hour profile of entrances and exits in the LTZ. In the examined day, 783 entrances and as many exits were identified. Relating this number of entrances to the number of entrances measured in the same day by the Automated Access Control System (59898 entrances), a penetration rate of about 1.3 percent was further confirmed by experimental measurements [11].

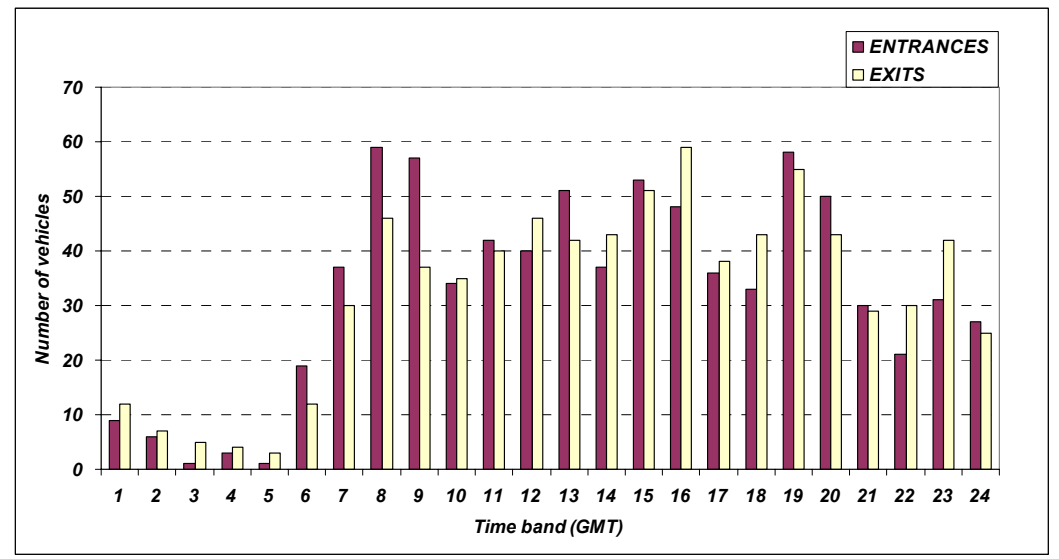

Figure 7: $\quad$ Entrances and exits of equipped vehicles in the LTZ zone.

\section{Conclusions}

The purpose of this paper is to present the potential benefits of massive FCD analysis for the evaluation process of urban traffic performances and travel patterns.

FCD is based on the collection of traffic data by locating the vehicles via GPS over the entire road network. This technique is a promising cost-effective solution to overcome some limitations from traditional fixed sensors chiefly due to their limited coverage and expensive costs of implementation and maintenance. 
At present FCD technique is not widely deployed and suffers from extremely small samples: the peculiarity of this study is indeed the type and the dimension of the fleet used to gather traffic data. It includes only private-owned vehicles and reaches a penetration rate of about 1.3 percent.

A full day of FCD related to the municipality area of Florence has been examined and a set of traffic performance measures has been estimated to give an insight on the practical potential of this technique to gain a better understanding of travel patterns and traffic performance in an urban area.

Further studies are currently underway to estimate origin-destination matrices by fusing traffic data from fixed sensors and FCD and to measure traffic performances along corridors and routes.

\section{Acknowledgement}

The authors would like to thank Octotelematics for kindly supplying the FCD used in this study.

\section{References}

[1] Cheu, R. L., Xie, C. \& Lee D., Probe Vehicles population and Sample Size for arterial speed Estimation, Computer-aided civil and infrastructure engineering, vol. 17(1), pp. 53-60, 2002.

[2] Hong, J., Zhang, X., Wei, Z., Li, L., Ren, Y., Spatial and Temporal Analysis of Probe Vehicle-based Sampling for Real-time Traffic Information System, Proceedings of the 2007 IEEE Intelligent Vehicles Symposium, Istanbul, Turkey, June 13-15, 2007.

[3] Srinivasan, Karthik, Jovanis, Determination of Number of Probe Vehicles Required for Reliable Travel Time Measurement in Urban Network, Transportation Research Record (1537), 15-22, 1996.

[4] Miwa, T., Tawada, Y., Yamamoto, T., Morikawa, T., En-Route Updating Methodology of Travel Time Prediction Using Accumulated Probe-Car Data, Proc. of the 11th ITS World Congress, Nagoya, Japan, 2004.

[5] Turksuma, S., The various uses of floating car data, Road Transport Information and Control, 2000 Tenth Intl. Conference on, Conf. Publ. No. 472), pp.51-55, April 2000.

[6] Yoon, J., Noble, B., Liu, M., Surface street traffic estimation, Proceedings of the 5th international conference on Mobile systems, applications and services, pp. $220-232,2007$.

[7] Kerner, B.S., Demir, C., Herrtwich, R.G., Klenov, S.L., Rehborn, H., Aleksic, M., Haug, A., Traffic state detection with floating car data in road networks, Proceedings of the 8th International IEEE Conference on Intelligent Transportation Systems, pp. 44-49.B.S., Sep, 2005.

[8] Li, Y., McDonald, M., Motorway incident detection using probe vehicles, Proceedings of the Institution of Civil Engineers Transport 158, Issue TR1, pages 11-15, February 2005. 
[9] Sethi, V., Bhandari, N., Koppleman, S., Schofer, J.L., Arterial incident detection using fixed detection and probe vehicle data, Transportation Research Part C, 3, No. 2, 99-112, 1995.

[10] Eisenman, S. M., List, G.F., Using Probe Data to Estimate OD Matrices, IEEE Intelligent Transportation Systems Conference, Washington. D.C., USA, October 36, 2004.

[11] Octotelematics s.r.l., www.octotelematics.it

[12] Demographic Report 2008, data from ISTAT, www.istat.it

[13] Comune di Firenze, www.comune.fi.it/home.htm

[14] Firenze on Wikipedia, http://it.wikipedia.org/wiki/Firenze;

[15] Istituto Superiore di Formazione e Ricerca per i Trasporti, http://www.isfort.it/ 
